\title{
CCAAT/Enhancer-Binding Protein Homologous Protein (CHOP) Deficiency Attenuates Heatstroke-Induced Intestinal Injury
}

\author{
Yan Cao ${ }^{1}$, Maiying Fan ${ }^{1}$, Yanfang Pei ${ }^{1}$, Lei $\mathrm{Su}^{2}$, Weiwei Xiao ${ }^{1}$, Fang $\mathrm{Chen}^{3}$, Jie Huang ${ }^{1}$, \\ Xiehong Liu ${ }^{3}$, Zhengtao Gu$^{4}$, Zhongwei Zhang ${ }^{1}$, Fangfang Yuan ${ }^{5}$, Yu Jiang ${ }^{3,6}$ and \\ Xiaotong Han $^{1,6}{ }_{(\mathbb{D}}$
}

Received 18 February 2021; accepted 4 October 2021

Abstract-The intestine is one of the main target organs involved in the pathological process of heatstroke. CCAAT/enhancer-binding protein homologous protein (CHOP) is involved in endoplasmic reticulum (ER) stress-induced apoptosis. This study aimed to explore the role of CHOP in heatstroke-induced intestinal injury and potential therapy. An in vitro heat stress (HS) model using Caco-2 cells was employed. We observed the role of CHOP in apoptosis-mediated intestinal epithelial cell injury secondary to HS by evaluating cell viability, lactate dehydrogenase release, apoptosis levels, and GRP78, PERK, ATF4, $\mathrm{CHOP}, \mathrm{Bcl}-2$, and BAX mRNA and protein expression. To further study the role of CHOP in HS-induced intestinal barrier dysfunction, we assessed transepithelial electrical resistance, paracellular tracer flux, ultrastructure of tight junctions, and protein expression of ZO-1 and occludin. Male wild-type mice and CHOP knockout mice were used for in vivo experiments. We evaluated serum d-lactate and diamine oxidase levels, histopathological changes, intestinal ultrastructure, and ZO-1 and occludin protein expression. HS activated the PERK-CHOP pathway and promoted apoptosis by upregulating BAX and downregulating Bcl-2; these effects were prevented by CHOP silencing. Intestinal epithelial barrier function was disrupted by $\mathrm{HS}$ in vitro and in vivo. CHOP silencing prevented intestinal

\footnotetext{
${ }^{1}$ Department of Emergency, Hunan Provincial People's Hospital, The First Affiliated Hospital of Hunan Normal University, Hunan Province, No.61 Western Jiefang Road, Changsha 410005, China ${ }^{2}$ Department of Intensive Care Medicine, General Hospital of Southern Theatre Command of PLA, Guangzhou, China ${ }^{3}$ Institute of Emergency Medicine, Hunan Provincial Key Laboratory of Emergency and Critical Care Metabonomics, Hunan Provincial People's Hospital, The First Affiliated Hospital of Hunan Normal University, Hunan Province, No.61 Western Jiefang Road, Changsha 410005, China

${ }^{4}$ Department of Treatment Center For Traumatic Injuries, The Third Affiliated Hospital of Southern Medical University, Guangzhou, China
}

\footnotetext{
${ }^{5}$ Department of Hematology, The Third Xiangya Hospital of Central South University, Changsha, China

${ }^{6}$ To whom correspondence should be addressed at Department of Emergency, Hunan Provincial People's Hospital, The First Affiliated Hospital of Hunan Normal University, Hunan Province, No.61 Western Jiefang Road, Changsha, 410005, China. Email: xiaotong_han@sina.com and Institute of Emergency Medicine, Hunan Provincial Key Laboratory of Emergency and Critical Care Metabonomics, Hunan Provincial People's Hospital, The First Affiliated Hospital of Hunan Normal University, Hunan Province, No.61 Western Jiefang Road, Changsha, 410005, China. Email: jiangyu@ @unnu.edu.cn
} 
barrier dysfunction in Caco-2 cells, whereas CHOP knockout mice exhibited decreased intestinal mucosal injury. The ER stress inhibitor 4-phenylbutyrate (4-PBA) prevented HSinduced intestinal injury in vitro and in vivo. This study indicated that CHOP deficiency attenuates heatstroke-induced intestinal injury and may contribute to the identification of a novel therapy against heatstroke associated with the ER stress pathway.

KEY WORDS: endoplasmic reticulum stress; heatstroke; CHOP; intestinal injury; 4-phenylbutyrate.

\section{INTRODUCTION}

With global warming, the morbidity and mortality of heatstroke have increased in recent years [1,2]. Clinically, heatstroke is characterized by extreme hyperthermia (core temperature $\geq 40{ }^{\circ} \mathrm{C}$ ), central nervous system dysfunction, and multiple organ failure. The intestine is one of the primary target organs involved in the pathophysiological process of heatstroke. The intestine is considered to be the largest reservoir of bacteria and toxins in the body. Approximately 300-500 different types of bacteria are present in the human intestine [3]. Endotoxins and pathogens can enter the circulatory system of the body through an impaired intestinal barrier, inducing endotoxemia and multiple organ dysfunction syndrome (MODS) [4]. However, the underlying mechanisms of intestinal injury in heatstroke remain poorly understood.

The mechanical barrier of the intestine is composed of intestinal epithelial cells, tight junctions (TJs), and a mucus layer covering the epithelial cell surface [5]. The intestinal mucosal epithelium provides a vital barrier against luminal pathogens. Endoplasmic reticulum (ER) is an important intracellular organelle that plays a pivotal role in maintaining cellular homeostasis. Recently, it has been reported that an increase in markers of ER stress was observed in the intestinal epithelia of patients with active inflammatory bowel disease (IBD), indicating that ER stress is relevant to the pathogenesis of IBD [6]. However, little is known about the role of ER stress in mediating the intestinal epithelial barrier dysfunction caused by heatstroke.

Apoptosis is programmed cell death. The integrity of the intestinal structure depends on the balance of intestinal epithelial cell proliferation and apoptosis [7]. Prior studies have found that excessive apoptosis can damage the intestinal epithelial barrier, and the apoptosis index is positively correlated with permeability [8]. In recent years, ER stress has become a hot topic in the field of apoptosis. CCAAT/enhancer-binding protein homologous protein (CHOP), one of the most sensitive factors involved in the regulation of ER stress, is a pivotal marker of ER stress-mediated apoptosis [9]. The upstream regulators of CHOP are three ER transmembrane proteins involving protein kinase R-like endoplasmic reticulum kinase (PERK), activating transcription factor 6 (ATF6) and inositol-required enzyme-1 $\alpha$ (IRE1 $\alpha)[9]$. Following the dissociation of glucose-regulated protein 78 (GRP78) from these three transmembrane proteins, three types of unfolded protein responses (UPRs) are triggered by ER stress. Among these pathways, the PERK/eukaryotic translation initiation factor $2 \alpha /$ activating transcription factor4 (PERK/ eIF2a/ATF4) signaling pathway is more important for CHOP protein expression compared with the other two pathways $[10,11]$. Thus, we hypothesized that CHOP may represent a key factor in regulating heatstrokeinduced intestinal barrier dysfunction.

4-Phenylbutyrate (4-PBA), an inhibitor of ER stress, is currently approved by the Food and Drug Administration (FDA) for the treatment of patients with urea cycle disorders (UCDs). 4-PBA functions as a chemical chaperone that prevents misfolded protein aggregation and relieves ER stress [12]. Recent studies have explored the potential therapeutic effects of 4-PBA in various experiments. For instance, in lipopolysaccharide (LPS)-induced acute lung injury (ALI) models, 4-PBA plays a protective role by inhibiting ER stress and autophagy [13]. In a mouse model of alcoholic hepatitis, 4-PBA prevented CHOP upregulation and inflammasome activation in the proximal small intestine [14]. The potential effect of 4-PBA on heatstroke and the underlying mechanisms require further study.

Therefore, our experiment was designed to investigate the role of CHOP in the pathogenesis of intestinal barrier dysfunction in the context of heatstroke and the therapeutic effect of the ER stress inhibitor 4-PBA. We applied two wellestablished models by performing heat stress (HS) on Caco-2 cells and mice to mimic heatstroke in vitro and in vivo. 


\section{MATERIALS AND METHODS}

\section{Cell Culture and Groups}

In our study, tunicamycin (TM) was used as an ER stress inducer [15]. Human gut-derived Caco-2 cells (Procell Life Science \& Technology Co., Ltd., Wuhan, China) were used as a model. These cells have been approved as suitable for studying intestinal epithelial barrier function. The cells were cultured in Dulbecco's modified Eagle's medium (DMEM) supplemented with $10 \%$ fetal bovine serum (FBS) at $37^{\circ} \mathrm{C}$ in a humidified atmosphere of $5 \% \mathrm{CO}_{2}$. At approximately $80 \%$ confluence, the cells were divided into the following eight groups: control group, HS group, CHOP-siRNA + HS group, control-siRNA + HS group, CHOP-plasmid + HS group, control-plasmid + HS group, 4-PBA + HS group, and $\mathrm{TM}+\mathrm{HS}$ group.

\section{Cell Treatment}

Caco- 2 cells were divided into the 8 groups as mentioned above. In the control group, the cells were grown in an incubator at $37^{\circ} \mathrm{C}$. In the HS group, the cells were grown in an incubator at $43{ }^{\circ} \mathrm{C}$ for $2 \mathrm{~h}$. In addition, the culture medium was refreshed, and the cells were further incubated at $37^{\circ} \mathrm{C}$ for an additional $6 \mathrm{~h}$. A previous study showed that the peak of cell injury and apoptosis was achieved $6 \mathrm{~h}$ after HS [16]. In the CHOP-siRNA + HS group, the cells were transfected with CHOP-siRNA $48 \mathrm{~h}$ before HS. In the control-siRNA + HS group, the cells were transfected with a control-siRNA $48 \mathrm{~h}$ before HS. In the CHOP-plasmid + HS group, the cells were transfected with a CHOP overexpression plasmid $48 \mathrm{~h}$ before HS. In the control-plasmid + HS group, the cells were transfected with a control plasmid $48 \mathrm{~h}$ before HS. In the 4-PBA + HS group, the cells were pretreated with $5 \mathrm{mmol} / \mathrm{L} 4-\mathrm{PBA} 1 \mathrm{~h}$ before exposure to HS. Finally, in the TM+HS group, the cells were pretreated with $5 \mu \mathrm{g} / \mathrm{ml} \mathrm{TM} 1 \mathrm{~h}$ before exposure to HS.

\section{Cell Transfection}

Caco-2 cells were transfected with CHOPsiRNA (GenePharma, Shanghai, China) or the CHOP overexpression plasmid (Tsingke, Beijing, China) according to the manufacturer's instructions. The cells were seeded in 6-well plates $24 \mathrm{~h}$ before transfection in antibiotic-free medium. The cell confluence rate before transfection was approximately $70 \%$. Then, $5 \mu$ of CHOPsiRNA, $5 \mu \mathrm{l}$ of control siRNA, $5 \mu \mathrm{l}$ of the CHOP overexpression plasmid, or $5 \mu \mathrm{l}$ of control plasmid was diluted in $250 \mu \mathrm{l}$ of Opti-MEM (Gibco, Carlsbad, CA, USA). Five microliters of Lipofectamine 2000 (Invitrogen, Carlsbad, CA, USA) was also diluted in $250 \mu 1$ of Opti-MEM for each reaction. The siRNA or plasmid solution was mixed gently with Lipofectamine 2000 and incubated for $20 \mathrm{~min}$ at room temperature. Then, the transfection complexes were added to 6-well plate cells at $500 \mu \mathrm{l}$ per well. The culture medium was refreshed after culture for $6 \mathrm{~h}$ at $37^{\circ} \mathrm{C}$ in a $5 \% \mathrm{CO}_{2}$ incubator.

The sense sequences ( $5^{\prime}$ to $\left.3^{\prime}\right)$ were as follows: CHOP sense, GCU GAG UCA UUG CCU UUC UTT and CHOP antisense, AGA AAG GCA AUG ACU CAG CTT; negative control sense, UUC UCC GAA CGU GUC ACG UTT; and negative control antisense, ACG UGA CAC GUU CGG AGA ATT.

\section{Cell Viability Assays}

The cell survival rate of each group was determined using a 3-(4,5-dimethylthiazol-2-yl)-2,5-diphenyltetrazolium bromide (MTT) kit (Beyotime Co, Shanghai, China) according to the manufacturer's instructions.

\section{Lactate Dehydrogenase Analysis}

Lactate dehydrogenase (LDH) is released when the cell membrane is damaged. Thus, $\mathrm{LDH}$ release was used to assess cell damage. LDH enzymatic activity was detected using an LDH kit following the manufacturer's instructions (JianChen Co, Nanjing, China).

\section{Hoechst 33258 Staining}

Hoechst 33258 staining was performed to observe the morphological changes of cellular nuclei. The cells of each group were washed with PBS, fixed with $4 \%$ polyformaldehyde for $10 \mathrm{~min}$, and then stained with Hoechst 33258 (Beyotime, Shanghai, China) solution for $10 \mathrm{~min}$ at room temperature in the dark. Morphological changes in the nuclei were then examined under a fluorescence microscope (Eclipse Ti-SR, Nikon Corporation, Tokyo, Japan). Under fluorescence microscopy, the living cellular nuclei stained by Hoechst dye present diffuse and uniform fluorescence, whereas the nuclei of dead cells exhibit blue dense staining or fragmentary dense staining. 


\section{Flow Cytometry Analysis}

Caco-2 cell apoptosis in each group was measured by flow cytometry based on Annexin V FITC-propidine iodide (PI) double staining (Invitrogen, Carlsbad, CA, USA). According to the manufacturer's instructions, the cells were digested with trypsin without ethylenediaminetetraacetic acid (EDTA) and centrifuged to harvest. The harvested cell number was approximately $1 \times 10^{5}$ cells. Next, Annexin V-FITC/PI staining was performed according to the protocol provided with the kit (Invitrogen, Carlsbad, CA, USA). After staining, the proportion of apoptotic cells was analyzed by flow cytometry (BD, New Jersey, USA). The apoptotic rate was calculated as the percentage of early apoptotic cells in the lower right quadrant.

\section{qRT-PCR Analysis}

Total RNA was extracted from the Caco- 2 cells of each group using Trizol (Invitrogen, Carlsbad, CA, USA). RNA was transcribed into cDNA using a PrimeScriptTM RT Reagent Kit (Takara, Shiga, Japan). The PCR mixture $(20-\mu l$ final volume per reaction) was prepared. Amplification was performed by quantitative real-time PCR with the TB Green Premix Ex TaqTM II kit (Takara, Shiga, Japan). The primer sequences are reported in Table 1. Actin served as the endogenous reference gene to normalize the data.

\section{Transepithelial Electrical Resistance Measurement}

Caco- 2 cells were plated on collagen-coated membrane Transwell inserts ( $3-\mu \mathrm{m}$ pore size filters, Corning, USA). The transepithelial electrical resistance (TEER) of cells was measured with an electrical resistance system (EVOM, World Precision Instruments, Berlin, Germany). The TEER was calculated by normalizing to the initial values and was expressed as percentages of the initial resistance values.

\section{Paracellular Tracer Flux Assay}

In this assay, FITC-dextran $(2.5 \mathrm{mg} / \mathrm{mL}$, Sigma-Aldrich, USA) was added to the upper chamber $2 \mathrm{~h}$ before the experimental endpoint. After incubation, the fluorescence concentration of FITC-dextran in the medium of the lower chamber was measured by fluorescence at Ex $490 \mathrm{~nm} / \mathrm{Em} 520 \mathrm{~nm}$ (TecanGENios reader, Tecan Group Ltd., CH).

\section{Animals}

$\mathrm{CHOP}^{-/-}$mice on a C57BL/6 background were purchased from Model Organism (Shanghai, China). Considering that estrogen can increase survival during heatstroke by relieving inflammatory responses and cardiovascular dysfunction [17], only male mice were used in the study. Male wild-type (WT) and $\mathrm{CHOP}^{-/-}$mice (12 weeks old) were used. The animal experiments were approved by the Ethics Committee of Hunan Provincial People's Hospital, The First Affiliated Hospital of Hunan Normal University, Changsha, China. All animals were housed in a controlled environment at a constant temperature of $21 \pm 2{ }^{\circ} \mathrm{C}$ with a 12-h light/dark cycle.

\section{Heatstroke Procedures}

The mice were randomly divided into six groups: the WT + sham group $(n=6), \mathrm{CHOP}^{-/-}+$sham group $(n=6), \mathrm{WT}+\mathrm{PBA}+$ sham group $(n=6), \mathrm{WT}+\mathrm{HS}$ group $(n=6), \mathrm{CHOP}^{-1-}+\mathrm{HS}$ group $(n=6)$, and $\mathrm{WT}+\mathrm{PBA}+\mathrm{HS}$ group $(n=6)$. We applied HS to mice to mimic heatstroke in vivo. To generate the

Table 1 Primer Sequences (5'-3')

\begin{tabular}{lll}
\hline Gene & & Primer \\
\hline GRP78 & Forward & GAATTCCTCCTGCTCCTCGT \\
& Reverse & CAGCATCATTAACCATCCTTTCG \\
PERK & Forward & ACGATGAGACAGAGTTGCGAC \\
& Reverse & ATCCAAGGCAGCAATTCTCCC \\
eIF2a & Forward & AAGCATGCAGTCTCAGACCC \\
& Reverse & GTGGGGTCAAGCGCCTATTA \\
ATF4 & Forward & ACAAGACAGCAGCCACTA \\
& Reverse & CTTACGGACCTCTTCTATCAG \\
CHOP & Forward & GGAAACAGAGTGGTCATTCCC \\
& Reverse & CTGCTTGAGCCGTTCATTCTC \\
Bcl-2 & Forward & GGTGGGGTCATGTGTGTGG \\
& Reverse & CGGTTCAGGTACTCAGTCATCC \\
Bax & Forward & TCACTGAAGCGACTGATGTCCC \\
& Reverse & ACTCCCGCCACAAAGATGGTC \\
Actin & Forward & ACCCTGAAGTACCCCATCGAG \\
& Reverse & AGCACAGCCTGGATAGCAAC \\
\hline
\end{tabular}


a

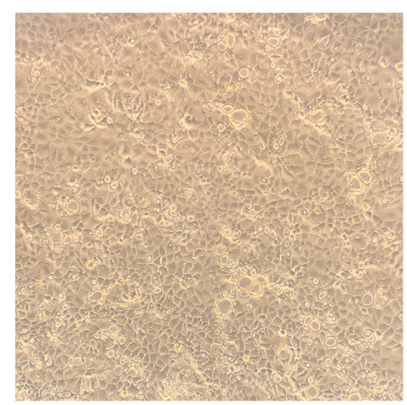

control

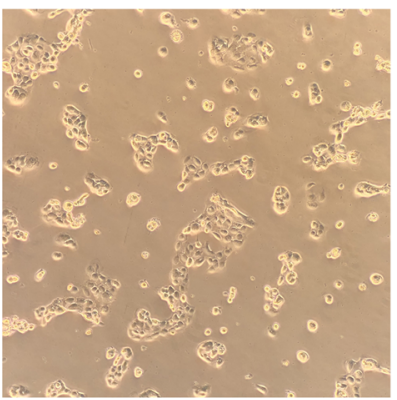

CHOP-plasmid+HS

b

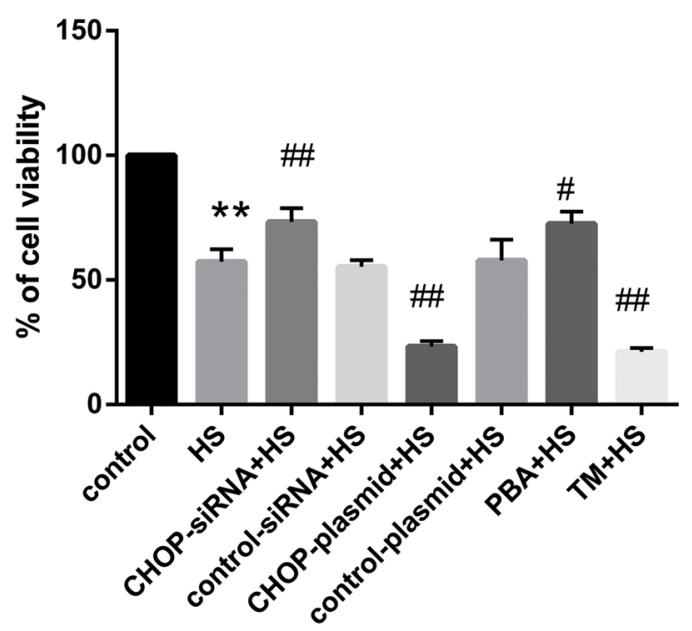

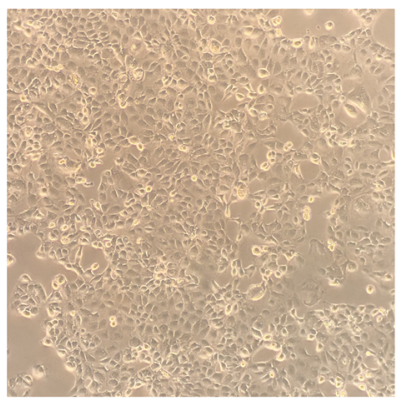

CHOP-SIRNA+HS

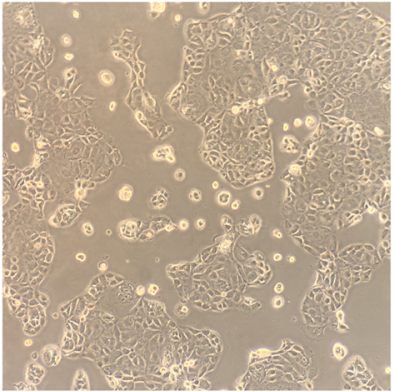

control-plasmid+HS

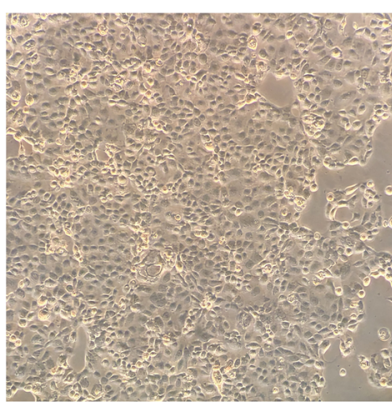

4-PBA+HS

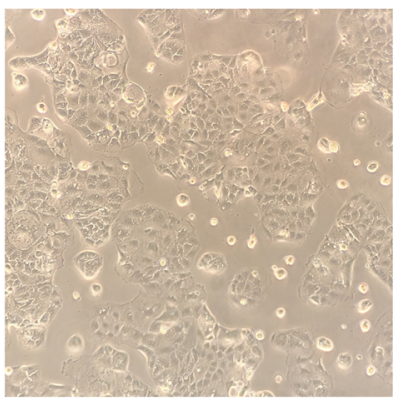

control-siRNA+HS

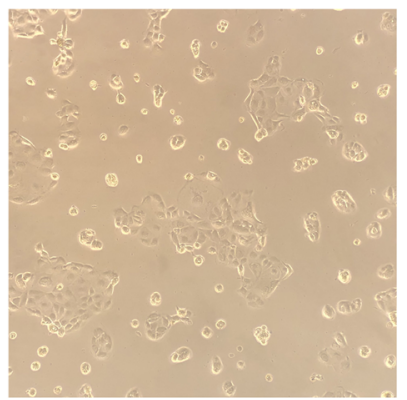

$\mathrm{TM}+\mathrm{HS}$
Fig. 1 Changes in cellular morphology, viability, and damage levels under HS in different experimental groups. a Morphological changes in Caco-2 cells as observed under a microscope (magnification $\times 100$ ). b MTT assay was used to detect cell viability. c LDH release analysis was used to detect the level of cell damage. Significant differences are indicated as follows: $* * P<0.01$ and $* P<0.05$ versus the control group; \#\#P<0.01 and $\# P<0.05$ versus the HS group.






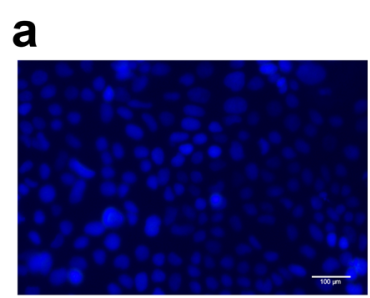

control

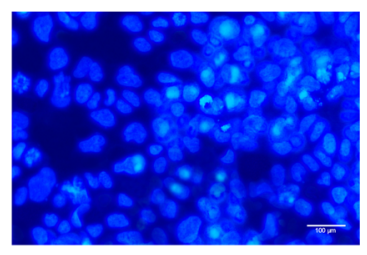

CHOP-plasmid+HS

b
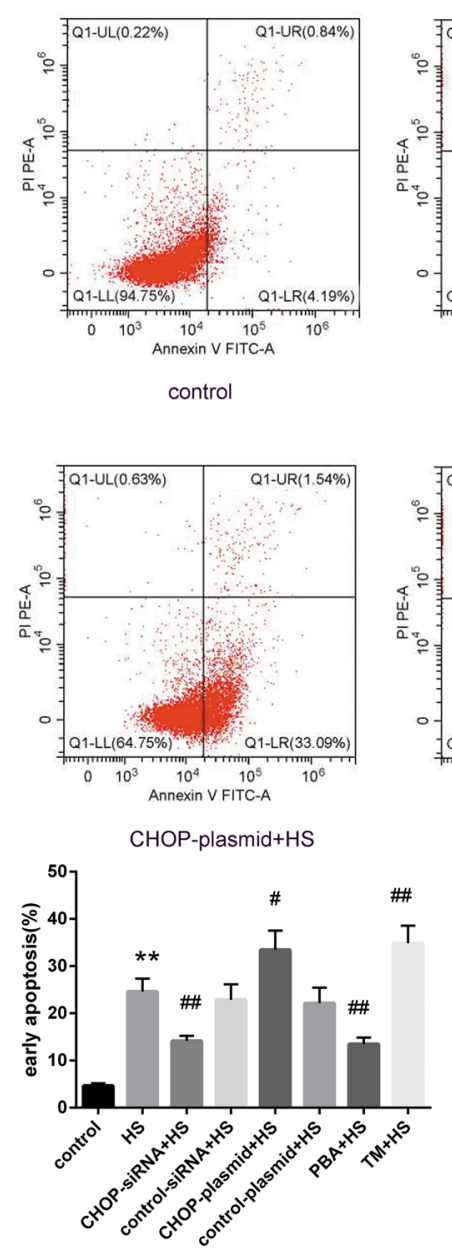

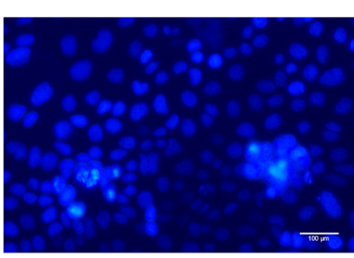

HS

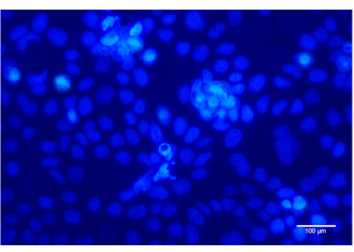

control-plasmid+HS

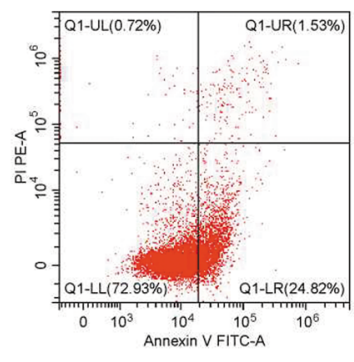

HS

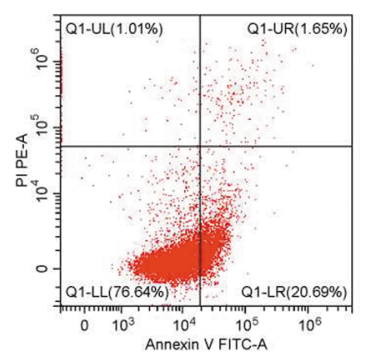

control-plasmid+HS

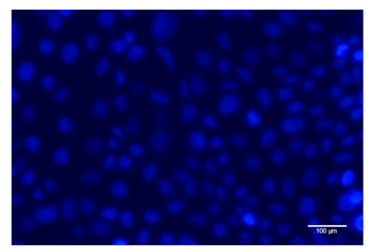

4-PBA+HS

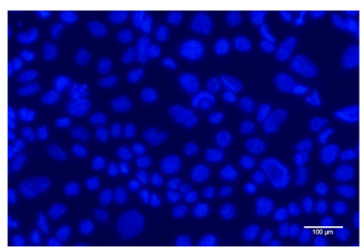

CHOP-SIRNA+HS

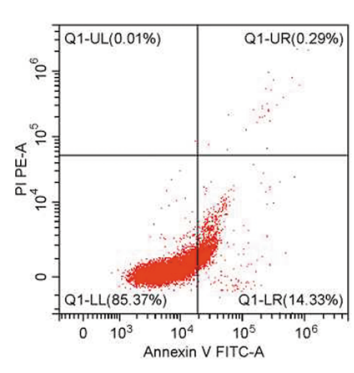

CHOP-SIRNA+HS

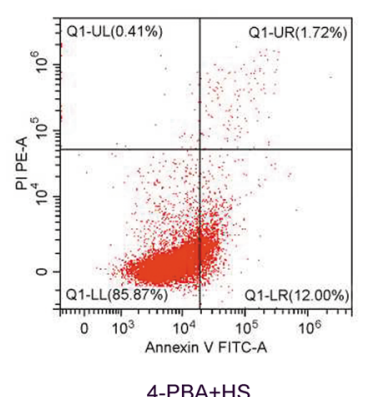

4-PBA+HS

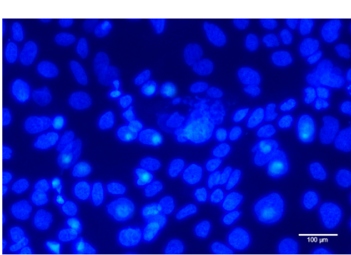

control-siRNA+HS

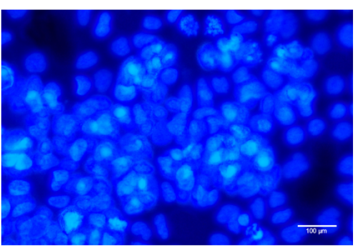

$\mathrm{TM}+\mathrm{HS}$

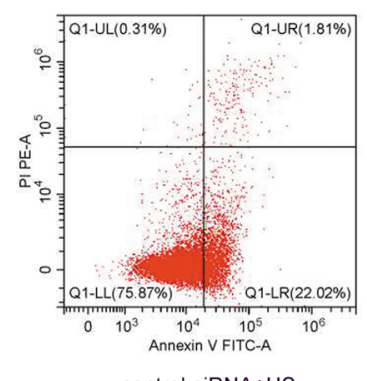

control-siRNA+HS

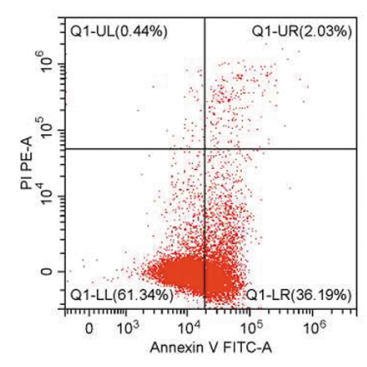

$\mathrm{TM}+\mathrm{HS}$ 
4 Fig. 2 Hoechst 33258 fluorescence nuclear staining and Annexin V-FITC/PI double-staining flow cytometry were used to detect the changes in apoptosis levels in different experimental groups. a Hoechst 33258 fluorescence nuclear staining was used to detect nuclear apoptotic morphological changes (magnification $\times 200$ ). b Annexin V-FITC/PI double-staining flow cytometry was used to detect the levels of apoptosis. Significant differences are indicated as follows: $* * P<0.01$ and $* P<0.05$ versus the control group; \#\#P<0.01 and $\# P<0.05$ versus the HS group.

heatstroke model, mice were transferred to an artificial climate chamber with an environmental temperature of $35.5 \pm 0.5{ }^{\circ} \mathrm{C}$ and humidity of $60 \pm 5 \%$. Rectal temperature $(\mathrm{Tc})$ was continuously measured using a mercury thermometer every $15 \mathrm{~min}$. The time point at which the Tc reached $42{ }^{\circ} \mathrm{C}$ was taken as the point of heatstroke onset $[18,19]$. After the Tc reached $42{ }^{\circ} \mathrm{C}$, the animals were allowed to recover at room temperature $\left(24 \pm 0.5^{\circ} \mathrm{C}\right)$. In the WT $+\mathrm{PBA}+\mathrm{HS}$ group, the WT mice were pretreated with 4-PBA (100 mg/kg, ip) before HS. In the $\mathrm{WT}+$ sham or $\mathrm{CHOP}^{-/-}+$sham group, the mice were maintained under sham-heated conditions at a temperature of $24 \pm 0.5^{\circ} \mathrm{C}$ and humidity of $35 \pm 5 \%$. The mice in the WT + PBA + sham group were pretreated with 4-PBA $(100 \mathrm{mg} / \mathrm{kg}$, ip) before being maintained under the same conditions. A previous study demonstrated that the average survival time of mice with heatstroke was approximately $6 \mathrm{~h}$ even when cooling treatment was applied [20]. Therefore, this time point was used for our subsequent experiment. The mice were sacrificed at this time point under anesthesia, and serum and the ileum were isolated.

\section{Serum d-Lactate and Diamine Oxidase Levels}

Serum samples were collected and measured using a corresponding enzyme-linked immunosorbent assay (ELISA) kit (Nanjing Jiancheng Co. Ltd., Nanjing, China) to detect serum d-lactate (D-LA) and diamine oxidase (DAO) following the manufacturer's instructions.

\section{Histopathology}

Ileum specimens from each group were fixed in $10 \%$ neutral-buffered formalin. The specimens were then embedded in paraffin blocks, sectioned at 5-7 $\mu \mathrm{m}$, and stained with hematoxylin and eosin (H\&E). The histopathological changes in ileal tissue were assessed under light microscopy.

\section{Ultrastructural Observation by Transmission Electron Microscopy}

The ultrastructural changes in Caco-2 cells or ileal tissues were observed by transmission electron microscopy. Caco-2 cell or ileum tissue specimens were fixed with $2.5 \%$ glutaraldehyde and then postfixed with $1 \%$ osmium tetroxide. Then, the specimens were dehydrated using a graded ethanol series (concentrations of $50 \%, 70 \%, 80 \%, 90 \%$, and $100 \%$ ) into pure acetone. After dehydration, the specimens were embedded with graded mixtures of acetone and SPI-PON812 resin and then polymerized for $12 \mathrm{~h}$ at $45{ }^{\circ} \mathrm{C}$ and $48 \mathrm{~h}$ at $60{ }^{\circ} \mathrm{C}$. Finally, ultrathin Sects. $(70 \mathrm{~nm})$ were stained and viewed under transmission electron microscopy (Hitachi, Tokyo, Japan).

\section{Western Blotting}

Proteins were extracted from Caco-2 cells or ileal tissues using RIPA buffer (Beyotime, Shanghai, China) to obtain total protein. The protein concentration was detected using a BCA protein assay kit (Applygen Technologies, Inc., Beijing, China). Equal amounts of protein $(30 \mu \mathrm{g})$ were separated on SDS-PAGE gels and transferred to nitrocellulose membranes (Millipore, Bedford, MA). The membranes were blocked in a 5\% skim milk-TBS solution at room temperature for $1 \mathrm{~h}$. Then, the membranes of cell proteins were incubated with diluted primary antibodies against the following proteins: GRP78, PERK, eIF $2 \alpha$, p-eIF $2 \alpha$, and CHOP (Cell Signaling Technology, Danvers, Massachusetts, USA); ATF4, B-cell lymphoma-2 (Bcl-2), and Bcl-2 Associated X Protein (Bax) (Proteintech Group, Inc. Rosemont, Illinois, USA); and zonula occluden-1 (ZO1) and occludin (Abcam, Massachusetts, United States). The membranes of tissue proteins were incubated with diluted primary antibodies against ZO-1 and occludin. Afterward, the membranes were washed thrice in PBS/ Tween-20 for 5 min with shaking. The membranes were then incubated with the secondary antibody (Zhongshan Inc., China) for $2 \mathrm{~h}$ at room temperature. The membranes were developed using a GE ImageQuant LAS 500 (GE Healthcare, USA). Quantification of the digitized images of the Western blot bands was performed using ImageJ software (National Institutes of Health, Bethesda, Maryland, USA). 
a
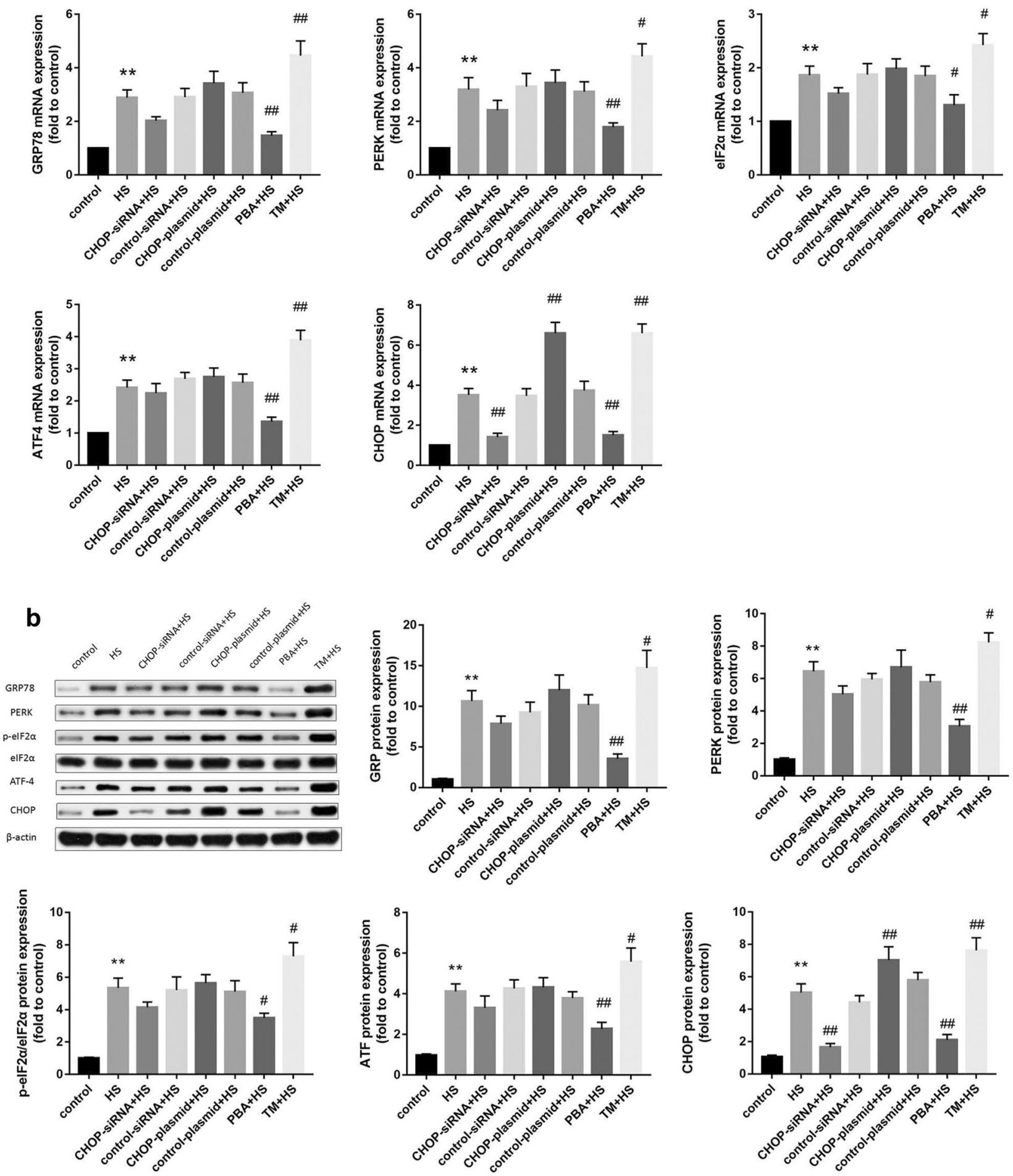
4 Fig. 3 a qRT-PCR was used to detect the mRNA expression of PERK-CHOP signaling pathway-related factors in different experimental groups. b Western blotting was used to detect GRP78, PERK, p-eIF2 $\alpha$, ATF4, and CHOP protein expression. The graphs show the relative band densities of the target protein to $\beta$-actin normalized against the control group. Significant differences are indicated as follows: $* * P<0.01$ and $* P<0.05$ versus the control group; \#\#P<0.01 and $\# P<0.05$ versus the HS group.

\section{Statistical Analysis}

All the data were presented as the mean \pm standard deviation, and differences were analyzed with oneway analysis of variance (ANOVA) among groups using SPSS statistical software (SPSS for Windows, version 19.0, Chicago, IL). A value of $P<0.05$ was considered statistically significant.

\section{RESULTS}

\section{The Effects of CHOP Silencing, CHOP Overexpression, an ER Stress Inhibitor, or an ER Stress Inducer on Caco-2 Cell Morphology, Viability and Damage Under HS}

An inverted microscope was used to observe the morphological changes of Caco-2 cells in each group. As shown in Fig. 1a, normal Caco-2 cells adhered to the wall and grew in a monolayer. HS induced cellular shrinkage, an increase in floating dead cells, and a decrease in living cells. After transfection with CHOPsiRNA or pretreatment with 4-PBA, the cell morphology was close to normal. Following transfection with the CHOP overexpression plasmid or pretreatment with $\mathrm{TM}$, cellular shrinkage was aggravated, and numerous floating dead cells were observed. To investigate the survival rate of Caco-2 cells after HS, we measured cell viability using the MTT assay. As shown in Fig. 1b, HS significantly decreased cell viability. After transfection with CHOP-siRNA or pretreatment with 4-PBA before HS, the cell viability increased compared with that of the HS group. Following transfection with the CHOP overexpression plasmid or pretreatment with $\mathrm{TM}$, cell survival significantly decreased compared with that of the HS group. Additionally, as shown in Fig. 1c, the LDH level was increased after HS. Transfection with CHOP-siRNA or pretreatment with 4-PBA decreased LDH levels after HS. However, the LDH level in the CHOP overexpression group or TM group was even greater than that in the HS group.
CHOP Activation Plays an Important Role in HS-Induced Apoptosis, and ER Stress Inhibition Can Alleviate HS-Induced Apoptosis

Morphological changes in apoptotic nuclei were observed by Hoechst 33258 staining, whereas apoptosis was detected by flow cytometry with Annexin V-FITC/ PI double staining. As shown in Fig. 2a, the nuclei of living cells stained by Hoechst exhibited diffuse and uniform fluorescence, while the nuclei of dead cells revealed condensed or fragmented staining. Compared with the control group, a large number of apoptotic nuclei with bright blue staining were present in the HS group. CHOP-siRNA or 4-PBA significantly prevented HS-induced apoptosis. In contrast, CHOP overexpression or TM further induced apoptosis.

As shown in Fig. 2b, the apoptosis level was detected using flow cytometry with Annexin V-FITC/ PI double staining. We observed that HS significantly increased Caco-2 cell apoptosis levels. In the CHOPsiRNA and 4-PBA groups, the number of apoptotic cells significantly decreased compared with the HS group. CHOP overexpression and TM pretreatment exacerbated the apoptosis induced by HS. These results indicate that CHOP activation plays a crucial role in HS-induced apoptosis and that the ER stress inhibitor 4-PBA has a remarkable protective effect on Caco-2 cells under HS.

\section{The Effects of CHOP Silencing, CHOP Overexpression, an ER Stress Inhibitor, or an ER Stress Inducer on mRNA and Protein Expression Levels of Components of the HS-Activated PERK-CHOP Pathway}

In our present study, we used qRT-PCR and Western blotting to confirm the role of CHOP in the PERKCHOP pathway. As shown in Fig. 3a, HS significantly increased GRP78, PERK, eIF2a, ATF4, and CHOP mRNA expression levels. As shown in Fig. 3b, GRP78, PERK, ATF4, and CHOP protein expression levels in the HS group were significantly increased compared with those in the control group. eIF2a phosphorylation was enhanced after HS, whereas total eIF2a protein levels showed no significant changes.

Additionally, CHOP-siRNA significantly reduced CHOP expression at both the mRNA and protein levels. Conversely, following CHOP overexpression plasmid transfection, a significant increase in CHOP mRNA and protein expression was detected. Interestingly, $\mathrm{CHOP}$ 

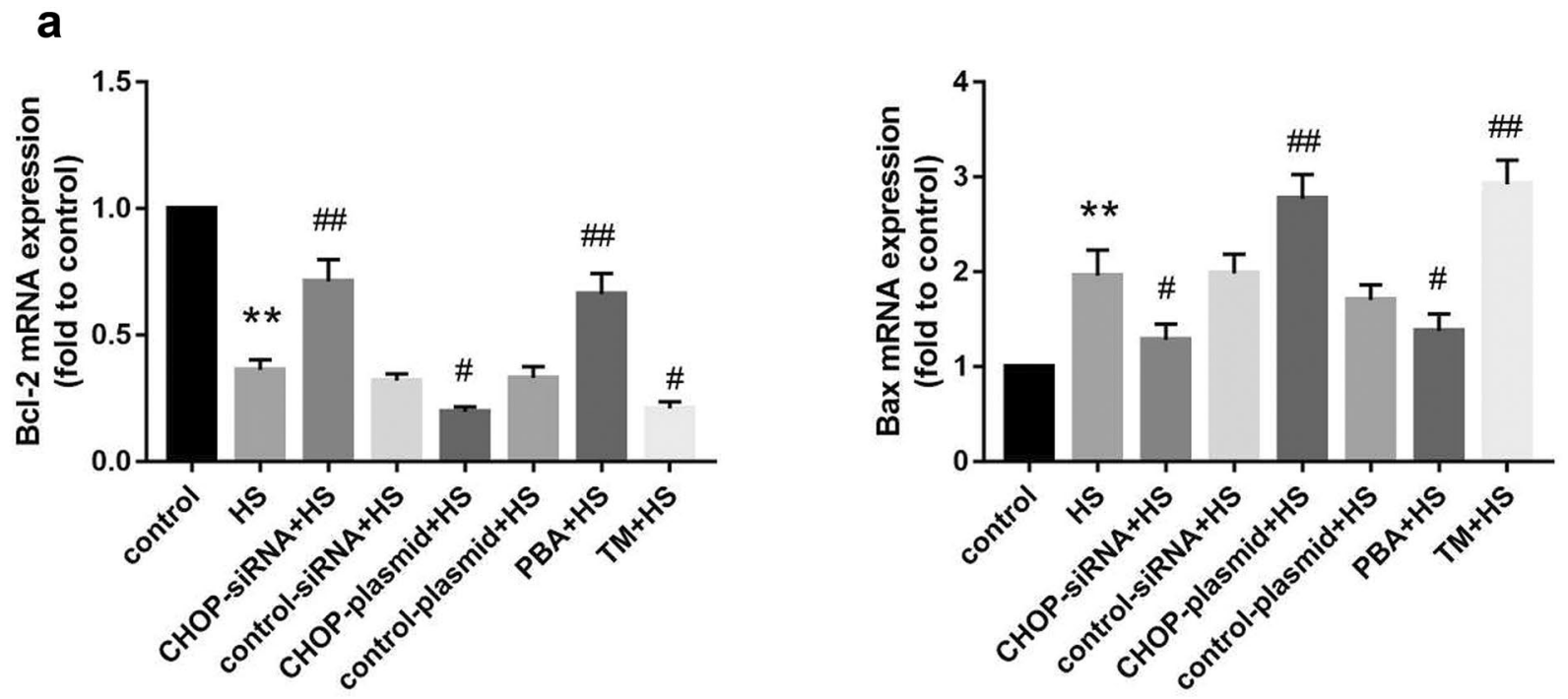

b
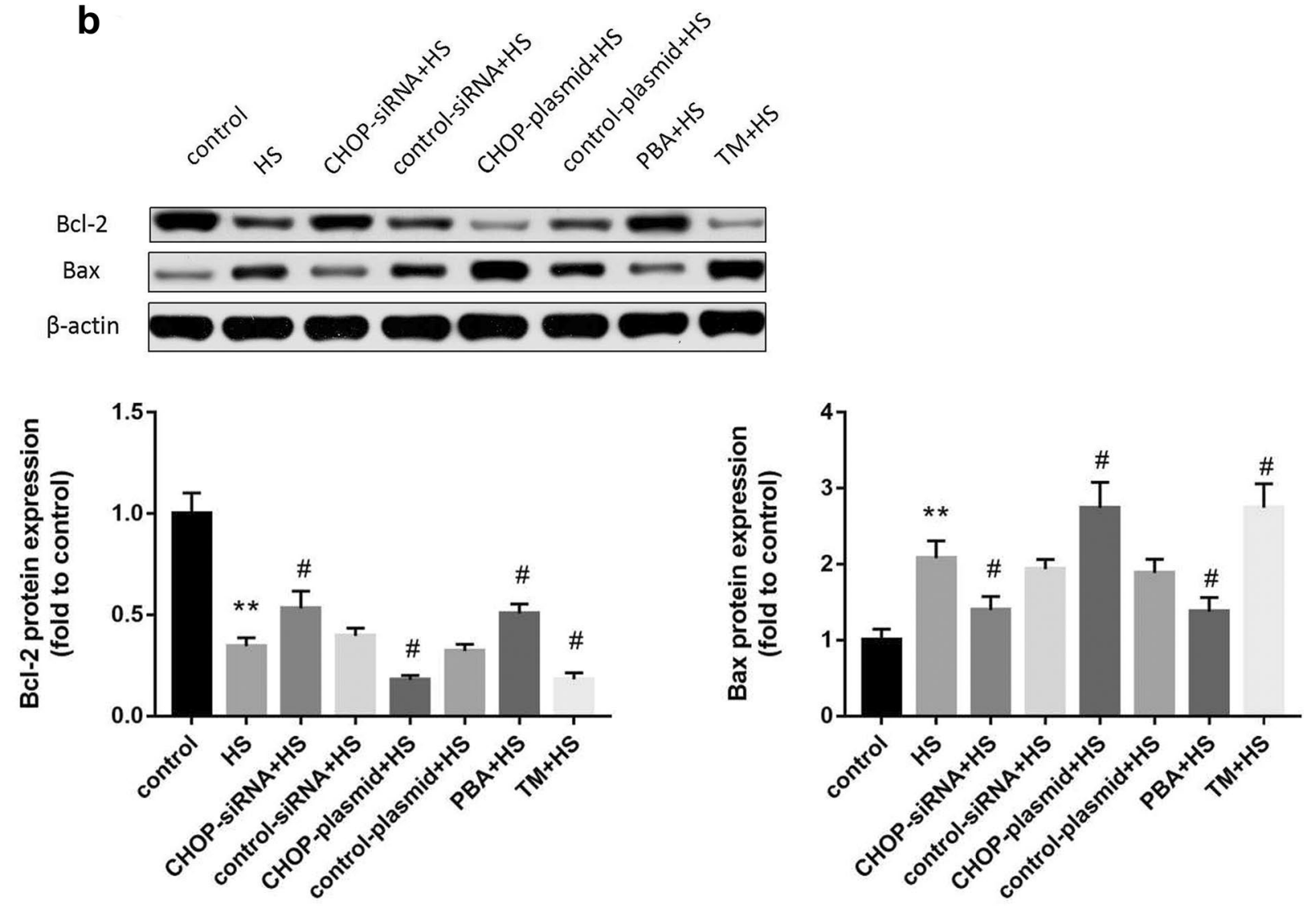

Fig. 4 a qRT-PCR was used to detect Bcl-2 and Bax mRNA expression in different experimental groups. b Western blotting was used to detect Bcl-2 and Bax protein expression. The graphs show the relative band densities of the target protein to $\beta$-actin normalized against the control group. Significant differences are indicated as follows: $* * P<0.01$ and $* P<0.05$ versus the control group; \#\#P<0.01 and \#P<0.05 versus the HS group. 
a
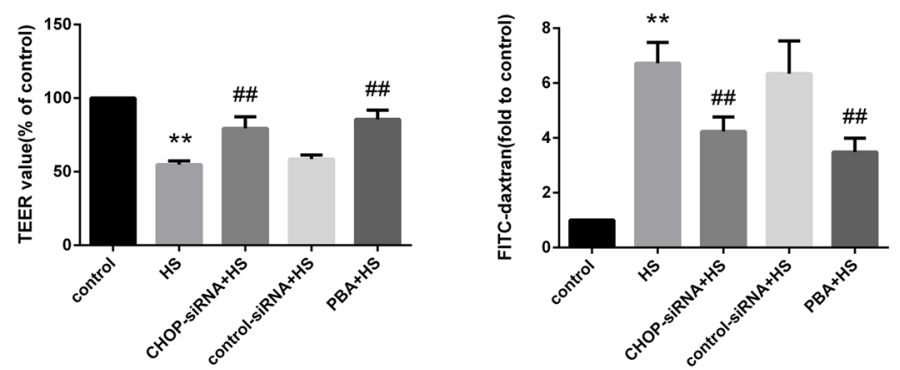

b

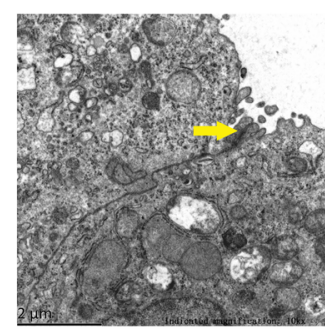

control

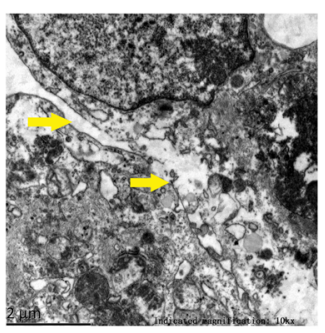

HS

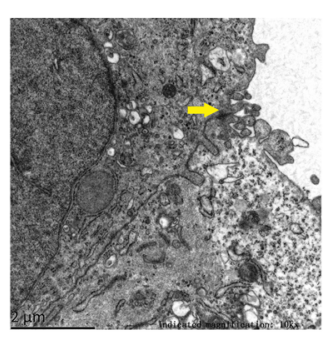

CHOP-SiRNA+HS

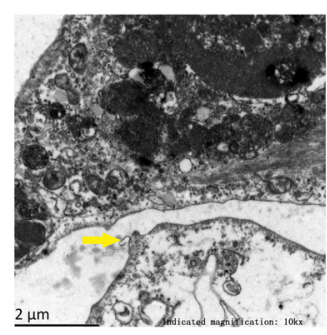

control-siRNA+HS

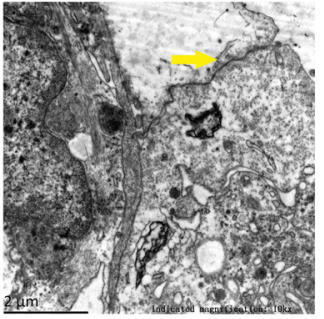

$\mathrm{PBA}+\mathrm{HS}$

C
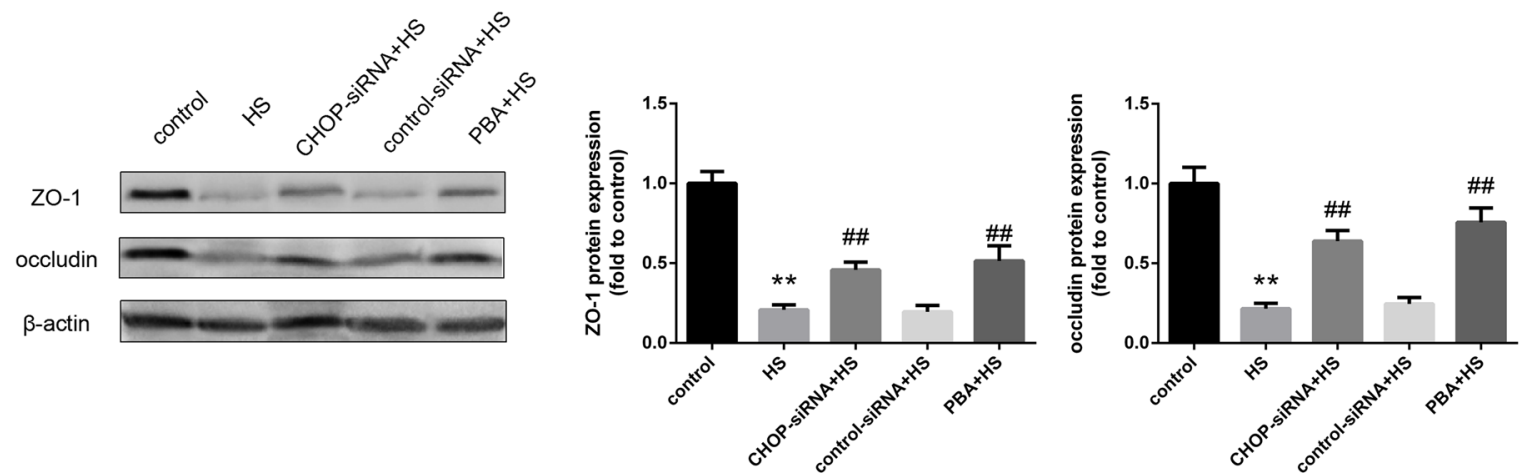

Fig. 5 Silencing CHOP by siRNA prevented intestinal barrier dysfunction induced by HS in Caco-2 cells. a TEER value is presented relative to the control group (\%TEER). The FITC-dextran value is presented relative to the control group. b Morphological ultrastructure of TJs under TEM. Yellow arrows indicate TJs (magnification $\times 10,000$ ). $\mathbf{c}$ Western blotting was used to detect ZO-1 and occludin protein expression. The graphs show the relative band densities of the target protein to $\beta$-actin normalized against the control group. Significant differences are indicated as follows: $* * P<0.01$ and $* P<0.05$ versus the control group; \#\#P<0.01 and $\# P<0.05$ versus the HS group.

silencing reduced GRP78, PERK, eIF2a, and ATF4 mRNA expression compared with that in the HS group, albeit without statistical significance. Subsequently, a reduction in GRP78, PERK, p-eIF2a, and ATF4 protein levels was also detected without statistical significance.

In addition, pretreatment with 4-PBA before HS significantly decreased the mRNA and protein expression of PERK-CHOP pathway members compared with that in the HS group, whereas pretreatment with TM before HS had the opposite effect, as shown in Fig. 3a, b.

\section{The Effects of CHOP Silencing, CHOP Overexpression, an ER Stress Inhibitor, or an ER Stress Inducer on Bcl-2 and Bax mRNA and Protein Expression}

Bcl-2 is an antiapoptotic protein, while Bax is a proapoptotic protein. To explore the role of CHOP in modulating Bcl-2 family members in response to HS, we used qRT-PCR and Western blotting to measure the mRNA and protein expression levels of Bcl-2 and 
a
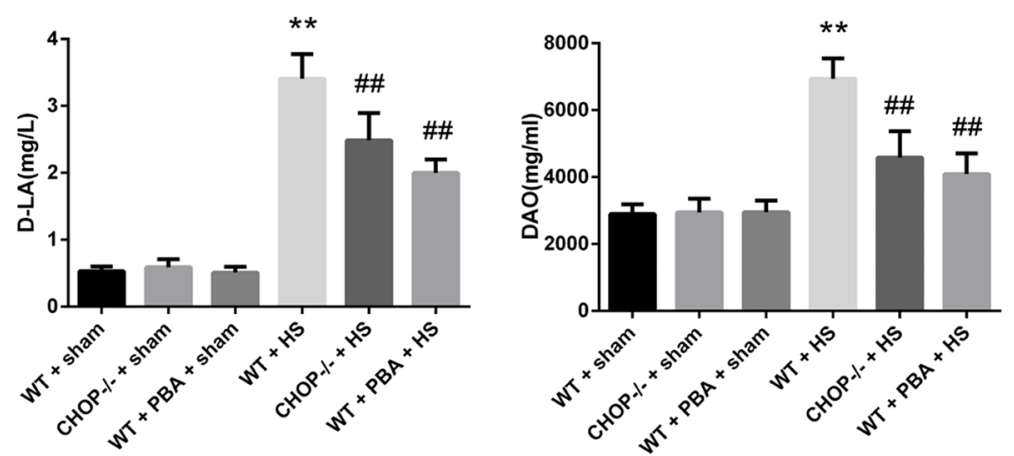

b

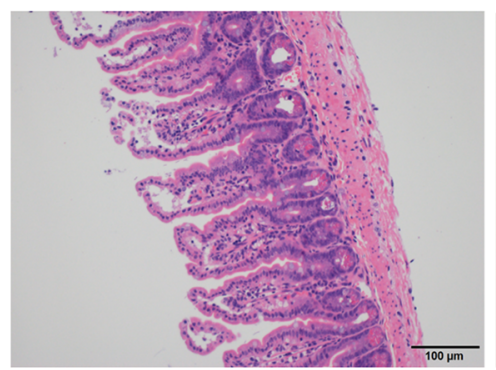

WT+sham

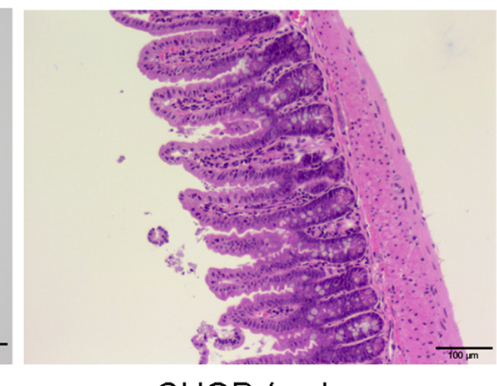

CHOP-I-+sham
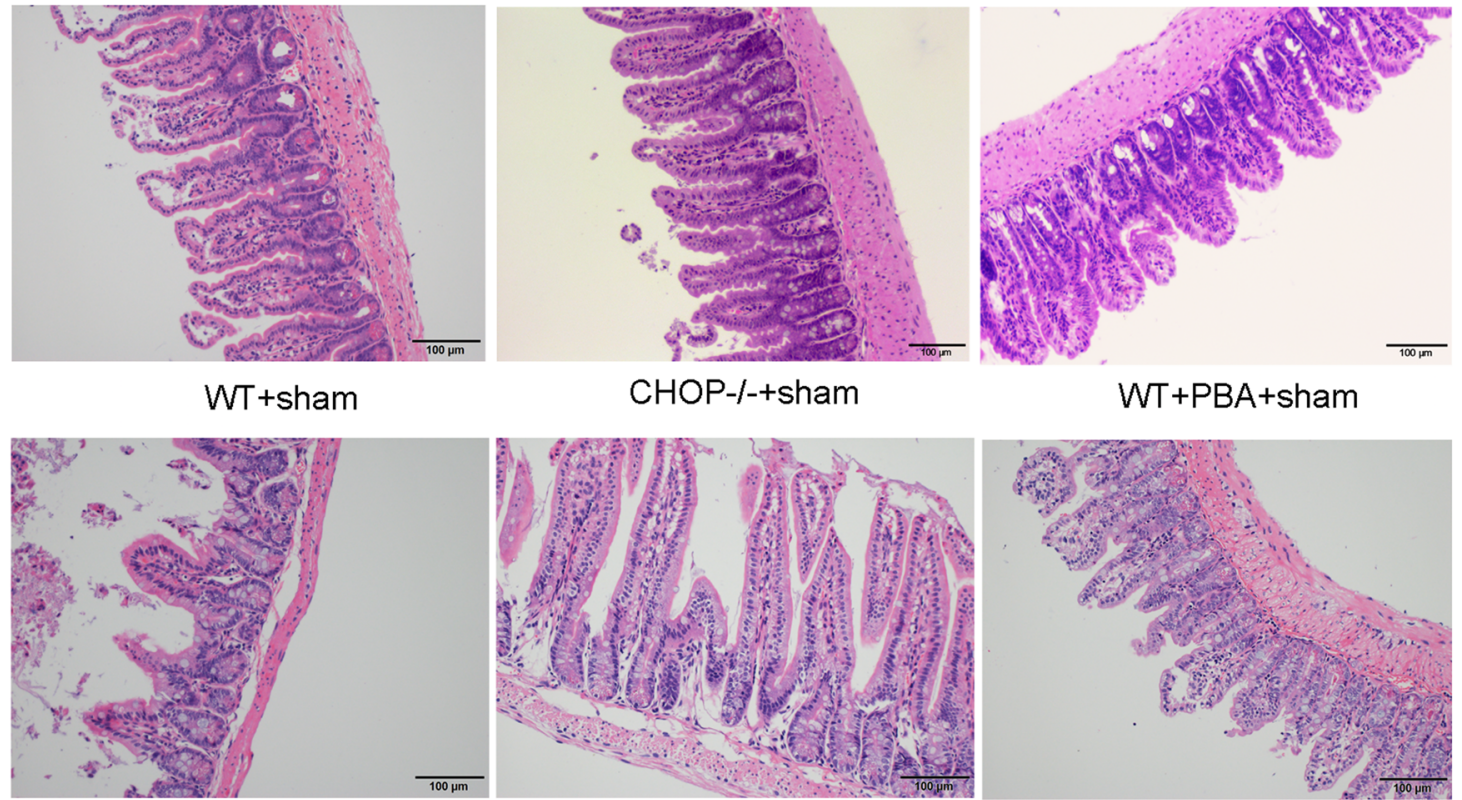

WT+HS

CHOP-l-+HS



$\mathrm{WT}+\mathrm{PBA}+\mathrm{HS}$

C
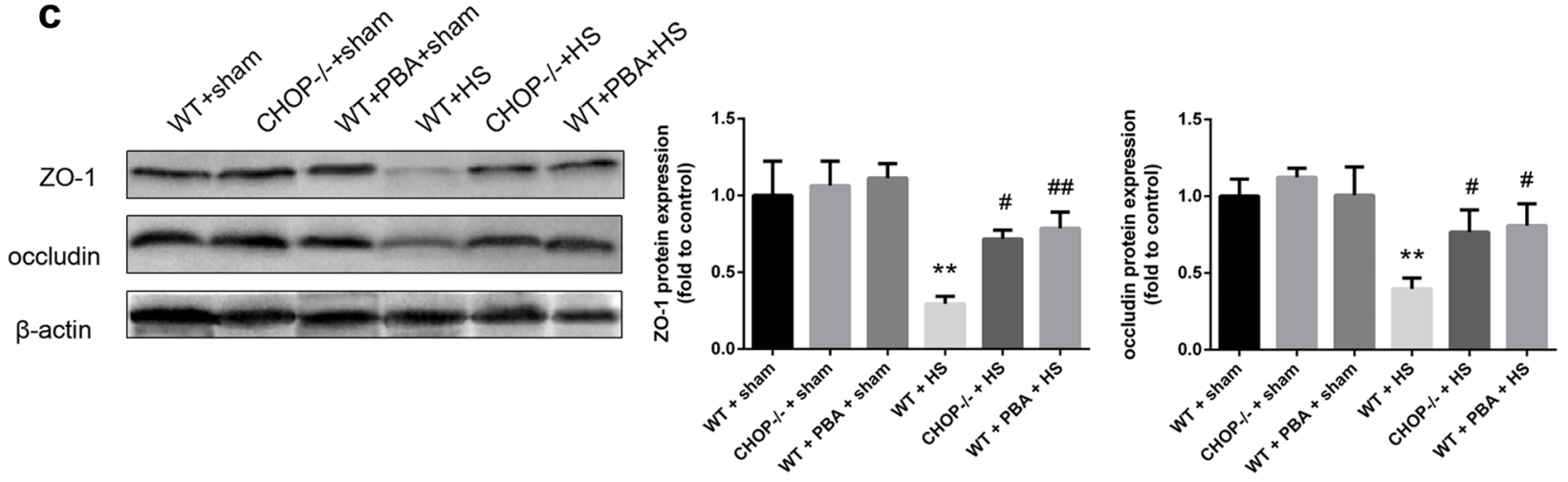
4 Fig. 6 CHOP knockout prevented intestinal barrier dysfunction induced by HS in mice. a Serum D-LA and DAO concentrations. b Histopathological changes in the ileum were observed by $\mathrm{H} \& \mathrm{E}$ staining (magnification $\times 200$ ). $\mathbf{c}$ Western blotting was used to detect ZO-1 and occludin protein expression. The graphs show the relative band densities of the target protein to $\beta$-actin normalized against the $\mathrm{WT}+$ sham group. Significant differences are indicated as follows: $* * P<0.01$ and $* P<0.05$ versus the $\mathrm{WT}+$ sham group; $\# \# P<0.01$ and $\# P<0.05$ versus the $\mathrm{WT}+\mathrm{HS}$ group.

Bax. As shown in Fig. 4a, b, Bax mRNA and protein expression levels in the HS group were significantly upregulated after $\mathrm{HS}$, whereas Bcl-2 mRNA and protein expression levels were markedly decreased. However, a significant reduction in Bax and an increase in Bcl-2 at both the mRNA and protein expression levels were observed when CHOP was silenced before HS. Following $\mathrm{CHOP}$ overexpression plasmid transfection before HS, a marked increase in Bax and a reduction in Bcl-2 mRNA and protein expression levels were detected. These findings suggested that $\mathrm{CHOP}$ is a key factor that mediates ER stress-induced apoptosis by modulating Bcl-2 family members in response to HS.

Furthermore, as shown in Fig. 4a, b, 4-PBA significantly increased Bcl-2 and decreased Bax mRNA and protein expression levels compared with the HS group. Pretreatment with TM before HS significantly decreased Bcl-2 and increased Bax the mRNA and protein expression levels compared with the HS group. These results demonstrated that the inhibition of ER stress has a protective effect on HS-induced apoptosis by upregulating Bcl-2 and downregulating Bax.

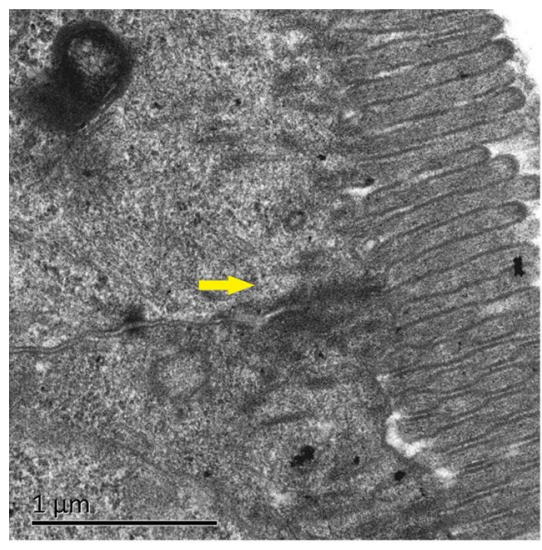

WT+sham

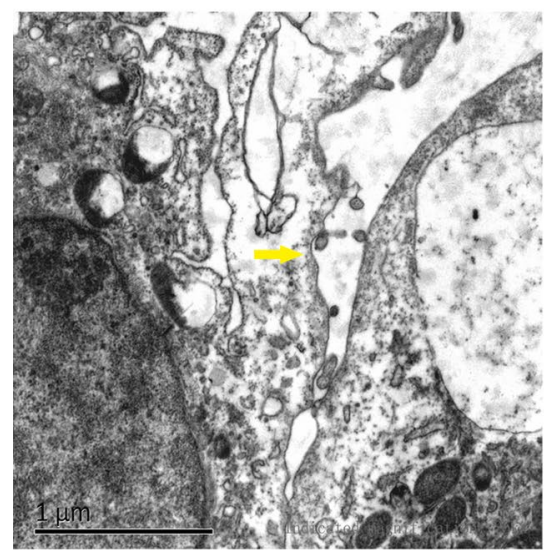

$\mathrm{WT}+\mathrm{HS}$

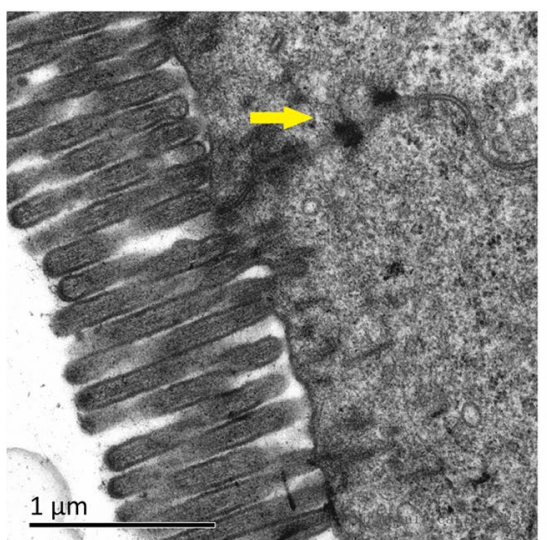

CHOP-I-+sham

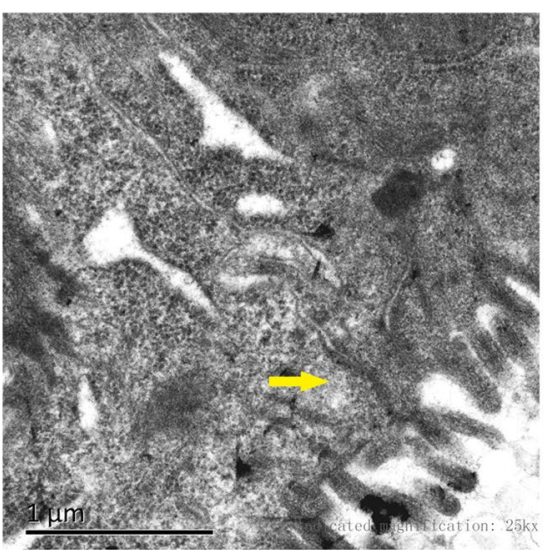

CHOP-/-+HS

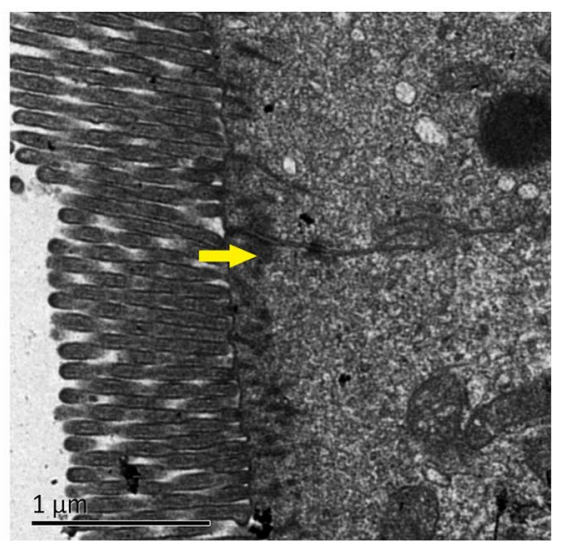

WT+PBA+sham

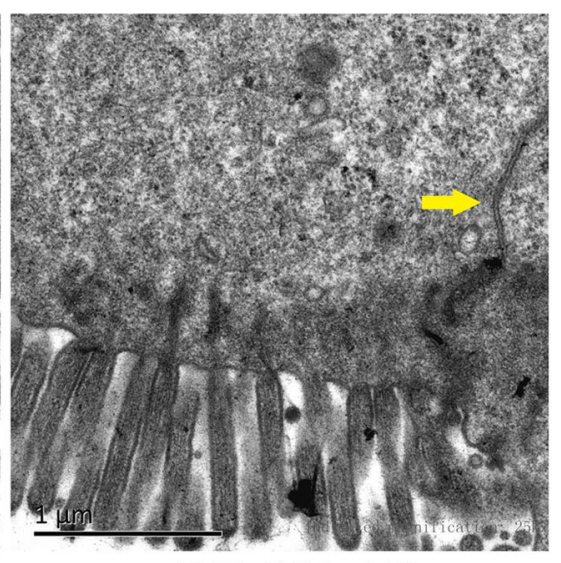

$\mathrm{WT}+\mathrm{PBA}+\mathrm{HS}$

Fig. 7 Morphological ultrastructure of TJs in mice. Yellow arrows indicate TJs (magnification $\times 25,000)$. 


\section{CHOP Deficiency or ER Stress Inhibition Prevents Intestinal Barrier Dysfunction Induced by HS Both In Vitro and In Vivo}

Increasing evidence has suggested that apoptosis is related to the increased expression of CHOP during ER stress [21]. In our current study, we identified that CHOP overexpression aggravates HS-induced apoptosis, whereas CHOP silencing or 4-PBA pretreatment protects against HS-induced apoptosis. Next, we investigated the role of CHOP silencing or 4-PBA pretreatment on intestinal barrier function in vitro and in vivo.

Epithelial barrier integrity and permeability of Caco-2 cells were measured by TEER and FITC-dextran flux. As shown in Fig. 5a, HS resulted in a significant reduction in TEER compared with the control group. CHOP silencing or 4-PBA pretreatment prevented the reduction in TEER induced by HS. Consistent with the TEER results, a significant increase in the paracellular permeability of FITC-dextran is noted in Fig. 5a, and CHOP silencing or 4-PBA pretreatment reversed the increase in paracellular permeability. As demonstrated by transmission electron microscopy in Fig. 5b, heat exposure destroyed the tight junction ultrastructure in Caco-2 monolayers. In the control group, intact tight junctions were noted between intestinal epithelial cells. In the HS group, cell and organelle structures were dramatically destroyed, the intercellular spaces were widened, and tight junctions were obviously opened. However, CHOP silencing or 4-PBA pretreatment greatly improved the ultrastructure of cells, and tight junctions were more continuous. As shown in Fig. 5c, Western blotting revealed that $\mathrm{ZO}-1$ and occludin protein expression decreased after HS, whereas CHOP silencing or 4-PBA pretreatment obviously increased ZO-1 and occludin expression.

In an in vivo study, increases in serum D-LA and DAO levels were observed in WT mice with heatstroke, and these increases were significantly inhibited in $\mathrm{CHOP}^{-/-}$mice or mice pretreated with 4-PBA, as shown in Fig. 6a. In addition, H\&E staining showed that mice exposed to HS presented profound damage to the epithelium of the small intestine, which manifested as extensive destruction of the villi and inflammatory cell infiltration, as shown in Fig. 6b. Heatstrokeinduced pathological changes were also significantly reduced in $\mathrm{CHOP}^{-/-}$mice pretreated with 4-PBA. As obtained from transmission electron microscopy in Fig. 7, we observed extreme destruction of tight junctions in the ileum of mice with heatstroke compared with the control tissue, which was significantly alleviated in $\mathrm{CHOP}^{-/-}$mice or upon pretreatment with 4-PBA. Next, we examined the expression levels of the tight junction proteins ZO-1 and occludin in the ileum. As shown in Fig. 6c, heatstroke decreased ZO-1 and occludin protein expression in WT mice, which was reversed in $\mathrm{CHOP}^{-/-}$mice or upon pretreatment with 4-PBA.

\section{DISCUSSION}

Intestinal barrier dysfunction plays a critical role in heatstroke progression, but the underlying mechanisms remain poorly understood. To our knowledge, the current study identifies a novel mechanism underlying heatstrokeinduced intestinal injury. Our results indicate that CHOP mediates intestinal epithelial apoptosis and barrier dysfunction induced by heatstroke. 4-PBA prevents apoptosis and improves the integrity of the intestinal barrier, underscoring its utility as a potential treatment for heatstroke.

As one of the key transcription factors in ER stress, CHOP is widely expressed in a variety of mammalian body cells. Under normal physiological conditions, CHOP expression is extremely low. However, $\mathrm{CHOP}$ expression is significantly increased in response to ER stress, thereby activating a series of downstream apoptotic molecules to induce apoptosis and participate in the occurrence and development of various pathological processes [21]. Apoptosis is a type of programmed cell death that is implicated in intestinal epithelial cell death [22]. The dynamic balance of intestinal epithelial cell proliferation and apoptosis maintains the homeostasis of the intestinal barrier. Under pathological conditions, repair and proliferation of the intestinal mucosa are difficult to achieve, and excessive apoptosis of intestinal mucosal cells will inevitably cause damage to the intestinal barrier [23]. In the pathophysiology of MODS secondary to heatstroke, the gastrointestinal tract is one of the first onset organs. Severe heatstroke can lead to increased intestinal epithelial apoptosis and impaired intestinal mucosal barrier followed by an endotoxemia-induced inflammation cascade and systemic inflammatory response syndrome (SIRS), which ultimately leads to MODS or even death [24]. In our study, we first established a cellular model of heatstroke using Caco- 2 cells and observed the role of CHOP in apoptosis-mediated intestinal epithelial injury. We observed that CHOP was activated in Caco- 2 cells 
after HS. Reductions in CHOP expression via transfection with siRNA increased cell viability and decreased apoptosis, whereas CHOP overexpression significantly decreased cell survival and increased apoptosis. These results revealed that CHOP was essential in HS-induced Caco-2 cell apoptosis.

CHOP is regulated by PERK, ATF6, and IRE1, but the PERK/eIF2a/ATF4 signaling pathway plays a more important role in CHOP activation compared with the other two UPR pathways [25]. In this pathway, PERK stimulates eIF2 $\alpha$ phosphorylation to enhance the translation of ATF4, thereby increasing CHOP transcription. Bcl-2 and Bax are downstream target genes of the PERK-CHOP apoptosis signaling pathway [26]. Our findings showed that HS increased the mRNA and protein expression of factors involved in the PERK-CHOP pathway in Caco-2 cells. By silencing CHOP before HS, Bax mRNA and protein expression levels were reduced, whereas Bcl-2 mRNA and protein expression levels were increased. However, $\mathrm{CHOP}$ overexpression upregulated Bax expression and downregulated Bcl-2 expression. Our data strongly support our hypothesis that CHOP plays a pivotal role in HS-induced apoptosis by regulating Bcl-2 and Bax.

Interestingly, after CHOP silencing, the expression of the ER stress chaperone proteins GRP78, PERK, eIF2a, and ATF4 was decreased, albeit without statistical significance. Similarly, in a study on the mechanism of rifampicin-associated liver damage, GRP78, PERK, and ATF4 expression was also downregulated after CHOP silencing [27]. Regarding the effect of silencing CHOP on GRP78 and the upstream factors of CHOP, such as PERK, eIF2a, and ATF4, previous studies have reported the presence of a feedback loop in the ER stress pathway [28]. Thus, we hypothesize that these upstream and downstream kinases may affect each other through a feedback loop in the same signaling pathway.

In addition, the results also indicated that pretreating Caco-2 cells with the ER stress inhibitor 4-PBA can significantly attenuate HS-induced changes in cell morphology and apoptosis by inhibiting PERK-CHOP pathways, subsequently downregulating the proapoptotic protein Bax and upregulating the antiapoptotic protein Bcl-2. In contrast, the ER stress-inducer TM aggravated Caco-2 cell apoptosis. Our results explore the potential therapeutic effects of 4-PBA in heatstroke.

Next, we explored the role of CHOP in regulating intestinal barrier function in vitro and in vivo. Maintenance of intestinal barrier integrity relies on a variety of mucosal structural components, such as tight junctions, which form zonula occludentes at the apical surface between cells [29]. It has been reported that extreme heat induces intestinal permeability and $\mathrm{TJ}$ protein disruption [30]. A previous study demonstrated that $\mathrm{CHOP}^{-/-}$mice are protected from bile duct ligation (BDL)-induced disruption of intestinal barrier function [31]. Our results showed that HS induced morphological injuries and ultrastructural damage (especially to TJs) of the intestinal mucosa, accompanied by an increase in intestinal permeability and disruption of epithelial integrity in vitro and in vivo. CHOP silencing significantly attenuated the HS-induced decrease in TEER and increase in FITC-dextran release in Caco- 2 cells by ameliorating the changes in tight junction structure and regulating TJ protein expression. In vivo studies with $\mathrm{CHOP}^{-/-}$mice further demonstrated that $\mathrm{CHOP}$ deficiency obviously alleviated HS-induced intestinal tissue damage and barrier dysfunction. In addition, our data showed that 4-PBA repaired dysfunction of the intestinal barrier following HS both in vitro and in vivo, indicating that ER plays a central role in the maintenance of intestinal mucosal homeostasis.

In conclusion, we have demonstrated for the first time that CHOP deficiency attenuates heatstroke-induced intestinal mucosal damage and barrier dysfunction. Here, we have provided strong evidence that targeting ER stress represents a novel therapeutic strategy for heatstroke.

\section{AUTHOR CONTRIBUTION}

Conceptualization, Yan Cao, Yu Jiang, and Xiaotong Han; methodology, Yan Cao and Zhengtao Gu; formal analysis, Yan Cao, Jie Huang, and Fangfang Yuan; investigation and data curation, Yan Cao, Xiehong Liu, and Zhongwei Zhang; writing-original draft preparation, Yao Cao, Maiying Fan, and Yanfang Pei; writingreview and editing, Lei Su, Weiwei Xiao, and Fang Chen; supervision and funding acquisition, Yan Cao. All the authors have read and agreed to the published version of the manuscript.

\section{FUNDING}

This research was funded by funds from the Natural Science Foundation of Hunan Province [2020JJ8082] and Hunan Provincial Health Commission Research Project [B2019066].

AVAILABILITY OF DATA AND MATERIAL 
All data generated or analyzed during the current study are included in this article.

\section{CODE AVAILABILITY}

No custom algorithm, software, or code was used in the present work.

\section{DECLARATIONS}

Ethics Approval and Consent to Participate Animal experiments in this study were approved by the ethical committee of Hunan Provincial People's Hospital.

Consent for Publication All the authors have read the manuscript and agreed to submit the paper to the journal.

Conflict of Interest The authors declare no competing interests.

OPEN ACCESS THIS ARTICLE IS LICENSED UNDER A CREATIVE COMMONS ATTRIBUTION 4.0 INTERNATIONAL LICENSE, WHICH PERMITS USE, SHARING, ADAPTATION, DISTRIBUTION AND REPRODUCTION IN ANY MEDIUM OR FORMAT, AS LONG AS YOU GIVE APPROPRIATE CREDIT TO THE ORIGINAL AUTHOR(S) AND THE SOURCE, PROVIDE A LINK TO THE CREATIVE COMMONS LICENCE, AND INDICATE IF CHANGES WERE MADE. THE IMAGES OR OTHER THIRD PARTY MATERIAL IN THIS ARTICLE ARE INCLUDED IN THE ARTICLE'S CREATIVE COMMONS LICENCE, UNLESS INDICATED OTHERWISE IN A CREDIT LINE TO THE MATERIAL. IF MATERIAL IS NOT INCLUDED IN THE ARTICLE'S CREATIVE COMMONS LICENCE AND YOUR INTENDED USE IS NOT PERMITTED BY STATUTORY REGULATION OR EXCEEDS THE PERMITTED USE, YOU WILL NEED TO OBTAIN PERMISSION DIRECTLY FROM THE COPYRIGHT HOLDER. TO VIEW A COPY OF THIS LICENCE, VISIT HTTP://CREATIVECOMMONS.ORG/LICENSES/BY/4.0/.

\section{REFERENCES}

1. Shimazaki, J., T. Hifumi, K. Shimizu, Y. Oda, and Y. Miyake. 2020. Clinical characteristics,prognostic factors, and outcomes of heat-related illness (heatstroke Study 2017-2018). Acute Medicine \& Surgery 7:e156 . https://doi.org/10.1002/ams2. 516.

2. Epstein, Y., and Y. Ran. 2019. Heatstroke. New England Journal Medicine 380: 2449-2459. https://doi.org/10.1056/NEJMra1810762.

3. Otani, S., and C.M. Coopersmith. 2019. Gut integrity in critical illness. Journal of Intensive Care 7: 17. https://doi.org/10.1186/ s40560-019-0372-6.

4. Snipe, R.M.J., A. Khoo, C.M. Kitic, P.R. Gibson, and R.J.S. Costa. 2018. The impact of exertional-heat stress on gastrointestinal integrity, gastrointestinal symptoms, systemic endotoxin and cytokine profile. European Journal of Applied Physiology 118: 389-400. https://doi.org/10.1007/s00421-017-3781-z.

5. Assimakopoulos, S.F., T. Christos, T. Konstantinos, F. Fotini, M. Ioannis, M. Markos, and C.A. Gogos. 2018. Gut-origin sepsis in the critically ill patient: pathophysiology and treatment. Infection 46:751-760. https://doi.org/10.1007/s15010-018-1178-5.

6. Coleman, O.I., and D. Haller. 2019. ER Stress and the UPR in Shaping Intestinal Tissue Homeostasis and Immunity. Frontiers in Immunology 10:2825. https://doi.org/10.3389/fimmu.2019.02825.

7. Parker, A., L. Vaux, A.M. Patterson, A. Modasia, D. Muraro, A.G. Fletcher, H.M. Byrne, P.K. Maini, A.J.M. Watson, and C. Pin. 2019. Elevated apoptosis impairs epithelial cell turnover and shortens villi in TNF-driven intestinal inflammation.Cell Death \& Disease 10:108. https://doi.org/10.1038/s41419-018-1275-5.

8. Joshua, L. Andersen, and Sally Kornbluth. 2013. The Tangled Circuitry of Metabolism and Apoptosis. Molecular Cell 49:399410. https://doi.org/10.1016/j.molcel.2012.12.026.

9. Iurlaro, R., and C. Munozpinedo. 2016. Cell death induced by endoplasmic reticulum stress. The FEBS Journal 283: 26402652. https://doi.org/10.1111/febs.13598.

10. Hu, H., M. Tian, C. Ding, and S. Yu. 2018. The C/EBP Homologous Protein (CHOP) Transcription Factor Functions in Endoplasmic Reticulum Stress-Induced Apoptosis and Microbial Infection. Frontiers in Immunology 9: 3083. https://doi.org/10.3389/fimmu. 2018.03083.

11. Li, Y., Y. Guo, J. Tang, J. Jiang, and Z. Chen. 2014. New insights into the roles of CHOP-induced apoptosis in ER stress. Acta Biochimica et Biophysica Sinica 46: 629-640. https://doi.org/10. 1093/abbs/gmu048.

12. Kolb, P., E.A. Ayaub, W. Zhou, V. Yum, J.G. Dickhout, and K. Ask. 2015. The therapeutic effects of 4-phenylbutyric acid in maintaining proteostasis. The International Journal of Biochemistry \& Cell Biology 61: 45-52. https://doi.org/10.1016/j.biocel. 2015.01.015.

13. Zeng, M., W. Sang, S. Chen, R. Chen, H. Zhang, F. Xue, Z. Li, Y. Liu, Y. Gong, and H. Zhang. 2017. 4-PBA inhibits LPS-induced inflammation through regulating ER stress and autophagy in acute lung injury models. Toxicology Letters 271: 26-37. https://doi.org/ 10.1016/j.toxlet.2017.02.023.

14. Gyongyosi, B., Y. Cho, P. Lowe, A. Iracheta-Vellve, A. Satishchandran, A. Ambade, and G. Szabo. 2019. Alcohol-induced IL-17A production in Paneth cells amplifies endoplasmic reticulum stress, apoptosis, and inflammasome-IL-18 activation in the proximal small intestine in mice. Mucosal Immunology 12(4):930-944. https://doi.org/10.1038/ s41385-019-0170-4. 
15. Yamamoto, K., and S. Ichikawa. 2019. Tunicamycin: chemical synthesis and biosynthesis. The Journal of Antibiotics 72:924933. https://doi.org/10.1038/s41429-019-0200-1.

16. Yi, G., L. Li, M. Luo, X. He, and L. Su. 2017. Heat stress induces intestinal injury through lysosome- and mitochondria-dependent pathway in vivo and in vitro. Oncotarget 8: 40741-40755.https:// doi.org/10.18632/oncotarget.16580.

17. Chen, S.H., F.M. Chang, K.C. Niu, M.Y. Lin, and M.T. Lin. 2006. Resuscitation from experimental heatstroke by estrogen therapy. Critical Care Medicine 34(4):1113-8. https://doi.org/10.1097/01. CCM.0000205756.04845.15

18. Liu, Z.F., J.J. Ji, D. Zheng, L. Su, and T.Q. Peng. 2019. Calpain-2 protects against heat stress-induced cardiomyocyte apoptosis and heart dysfunction by blocking p38 mitogen-activated protein kinase activation. J Cell Physiol 234 (7): 10761-10770. https:// doi.org/10.1002/jcp.27750.

19. Li, L., H. Tan, Z. Zou, J. Gong, J.J. Zhou, N. Peng, L. Su, M. Marc, D.Z. Cai, and Z.T. Gu. 2020. Preventing necroptosis by scavenging ROS production alleviates heat stress-induced intestinal injury. International Journal of Hyperthermia 37(1):517-530. https://doi. org/10.1080/02656736.2020.1763483.

20. Liu, Z., X. Sun, J. Tang, Y. Tang, H. Tong, Q. Wen, Y. Liu, and L. Su. 2011. Intestinal inflammation and tissue injury in response to heat stress and cooling treatment in mice. Molecular Medicine Reports 4(3):437-43.https://doi.org/10.3892/mmr.2011.461

21. Yao, Y., Q. Lu, Z. Hu, Y. Yu, Q. Chen, and Q.K. Wang. 2017. A non-canonical pathway regulates ER stress signaling and blocks ER stress-induced apoptosis and heart failure. Nature Communications 8: 133. https://doi.org/10.1038/s41467-017-00171-w.

22. Ji, X., W. Zheng, and W. Yao. 2019. Protective Role of Hydrogen Gas on Oxidative Damage and Apoptosis in Intestinal Porcine Epithelial Cells (IPEC-J2) Induced by Deoxynivalenol: A Preliminary Study.[J]. Toxins (Basel) 2019,12(1):5. https://doi.org/10. 3390/toxins 12010005 .

23. Gunther, C., H. Neumann, M.F. Neurath, and C. Becker. 2013. Apoptosis, necrosis and necroptosis: cell death regulation in the intestinal epithelium. Gut 62: 1062-1071. https://doi.org/10. 1136/gutjnl-2011-301364.

24. Yu, J., F. Liu, P. Yin, H. Zhao, W. Luan, X. Hou, Y. Zhong, D. Jia, J. Zan, and W. Ma. 2013. Involvement of oxidative stress and mitogen-activated protein kinase signaling pathways in heat stress-induced injury in the rat small intestine. Stress 16: 99-113. https://doi.org/10.3109/10253890.2012.680526.

25. Nishitoh, H. 2012. CHOP is a multifunctional transcription factor in the ER stress response. Journal of Biochemistry 151: 217-219. https://doi.org/10.1093/jb/mvr143.

26. Rozpedek, W., D. Pytel, B. Mucha, H. Leszczynska, J.A. Diehl, and I. Majsterek. 2016. The Role of the PERK/eIF2 $\alpha /$ ATF4/CHOP Signaling Pathway in Tumor Progression During Endoplasmic Reticulum Stress. Current Molecular Medicine 16: 533-544. https://doi.org/10.2174/1566524016666160523143937.

27. Zhang, W., L. Chen, Y. Shen, and J. Xu. 2016. Rifampicininduced injury in L02 cells is alleviated by 4-PBA via inhibition of the PERK-ATF4-CHOP pathway. Toxicol In Vitro $36: 186-196$. https://doi.org/10.1016/j.tiv.2016.07.017.

28. Ohoka, N., S. Yoshii, T. Hattori, and H. Hayashi. 2005. TRB3, a novel ER stress-inducible gene, is induced via ATF4-CHOP pathway and is involved in cell death. The EMBO Journal 24:1243-55. https://doi.org/10.1038/sj.emboj.7600596.

29. Odenwald, MA., and J.R. Turner. 2017. The intestinal epithelial barrier: a therapeutic target? Nature Reviews Gastroenterology \& Hepatology 14:9-21. https://doi.org/10.1038/nrgastro.2016.169.

30. Xiao, G., F. Yuan, Y. Geng, X.W. Qiu, and Z.F. Liu. 2015. Eicosapentaenoic acid enhances heatstroke-impaired intestinal epithelial barrier function in rats. Shock 44(4):348-56. https://doi.org/10. 1097/SHK.0000000000000417.

31. Liu, R., X. Li, Z. Huang, B.S. Ganesh, G. Lai, WM Pandak, P.B. Hylemon, J.S. Bajaj, and A.J. Sanyal. 2018. C/EBP homologous protein-induced loss of intestinal epithelial stemness contributes to bile duct ligation-induced cholestatic liver injury in mice. Hepatology 67(4):1441-1457. https://doi.org/10.1002/hep.29540.

Publisher's Note Springer Nature remains neutral with regard to jurisdictional claims in published maps and institutional affiliations. 